\title{
Thermopower of Molten Aluminium in Ultrasonic Field
}

\author{
L. Moraru* \\ Department of Physics, Faculty of Sciences, University of Galati \\ Str. Domneasca nr. 111, 6200 Galati, Romania \\ (Received February 24, 2003; revised version September 15, 2003) \\ Temperature dependence on the liquid aluminium thermopower is re- \\ ported for the range $933-1043 \mathrm{~K}$. In order to highlight the ultrasonic wave \\ effect on the thermoelectric power, the experiments were carried out under \\ similar conditions with and without sonication. The lower values of the ther- \\ mopower in sonication conditions are explained by the decrease in the melt \\ temperature gradient and due to the specific effects of ultrasound propaga- \\ tion process (acoustic flow and acoustic cavitation). A functional relationship \\ was found, $Q=-A / T+B T$, where $A$ and $B$ are positive constants. These \\ two terms are regarded as electron-ion collisions and spatial redistribution \\ of conduction electrons in the necessary temperature gradient. The effect \\ of cavitation at high temperature is debated. A good agreement between \\ the experimental data and theoretical models of the transport properties is \\ achieved.
}

PACS numbers: 72.15.Cz, 43.25.+y

\section{Introduction}

The electronic transport properties, particularly electrical resistivity and thermopower, of simple liquid metals and alloys have been studied with the Ziman nearly free-electron theory $[1,2]$ in conjunction with various model potentials (such as those of Heine and Abarenkov [3], Animalu and Heine [4] and Ashcroft [5]). The electrical resistivity values and thermopower of simple liquid metals calculated using these model potentials are in good agreement with the experimental data. Theoretical interpretation of transport processes in liquids has often been concentrated on those with particularly simple units of structure, such as molten metals. A systematic study for certain classes of melt could provide valuable diagnostic information about structure. Unfortunately, such information is still far from systematic.

*e-mail: Luminita.Moraru@ugal.ro 
In this report we present new experimental data concerning the thermopower $Q$ of liquid aluminium under ultrasonic irradiation above the melting point. This metal is the best electrical conductor with low resistivity and it is known to be the most nearly free-electron metal. Also, aluminium has a very low melting temperature [6].

The new and interesting finding from these results is that there appears to be a decrease in thermopower values due to the ultrasound effect.

Although these pseudopotential models have had remarkable success in predicting the electrical resistivity of the most simple liquid metals, there is no unitary theory of the transport coefficient modification phenomena under the influence of ultrasound. The present study may be the first attempt to deal with the effects of ultrasonic waves on thermopower of liquid metals. We start by presenting the measurements of the thermopower in absence of ultrasonic field. The thermopower of molten aluminium was determined from measurements of the temperature dependence of the electrical resistivity. Classical DC electrical resistivity measurements were made. The comparison between thermopower values obtained in the absence of ultrasound, and the values reported in several research works [1-5, 7-9] as well as in The Physical Properties of Liquid Metals (1993) [10] showed good agreement. Finally, we discuss how the ultrasonic field influences thermopower values.

Taking into account the relationship between the transport coefficients in the nearly free-electron approximation, the form of the thermopower is [7]:

$$
Q=\frac{\pi^{2} k_{\mathrm{B}}^{2} T}{3|e| \varepsilon_{\mathrm{F}}} \varepsilon_{\mathrm{F}}\left[\frac{\partial \ln \rho(\varepsilon)}{\partial \varepsilon}\right]_{\varepsilon=\varepsilon_{\mathrm{F}}}=\frac{\pi^{2} k_{\mathrm{B}}^{2} T}{3|e| \varepsilon_{\mathrm{F}}}\left[\frac{\partial[\ln \rho(\varepsilon)]}{\partial \ln \varepsilon}\right]_{\varepsilon=\varepsilon_{\mathrm{F}}}
$$

where $k_{\mathrm{B}}$ is Boltzmann's constant, $\varepsilon_{\mathrm{F}}$ is the Fermi energy, $T$ is the absolute temperature and $\rho$ is the electrical resistivity. The quantity enclosed in brackets is to be evaluated at $\varepsilon=\varepsilon_{\mathrm{F}}$. In spite of the general formalism for calculating the metals thermopower, detailed predictions of the sign, magnitude and temperature dependence of $Q$ have so far been obtained only for a hypothetical metal whose electrons behave as a free-electron gas.

At high temperature, the relationship between the thermopower for aluminium and the absolute temperature is [7]:

$$
Q=-\frac{A}{T}+B T
$$

where $A$ and $B$ are positive constants. The term proportional with $1 / T$ characterizes the electron-ion collisions, being associated with the transition probability of the electron, from one state to another. The term proportional with $T$ describes the contribution of a diffusion process, associated with the spatial electrons redistribution in temperature gradient field. In the presence of ultrasound in liquid metal mass, the thermopower values are decreasing. 


\section{Experimental method}

The samples used in this study were $99.97 \mathrm{wt} \% \mathrm{Al}$, electrolytically refined, with impurities: $\mathrm{Fe}(<80 \mathrm{ppm}), \mathrm{Si}(<80 \mathrm{ppm}), \mathrm{Cu}(<30 \mathrm{ppm})$ and Zn $(<30 \mathrm{ppm})$.

The molten metal sample is put into a ceramic crucible, located inside the electrical furnace supplied by the power supplier. Measurements of the furnace temperature heats were made with a $\mathrm{Pt} / \mathrm{Pt}-13 \% \mathrm{Rh}$ thermocouple inserted through the furnace wall. The temperature inside the crucible was separately determined by two chromel-alumel thermocouples encased in a quartz sheath of $1.7 \mathrm{~mm}$ (outer diameter) placed at various locations on the central axis of the crucible. The thermocouple moved along the axis by a micrometer attached to the base of the apparatus. Any errors in the measured absolute temperature inside the crucible are small and do not affect the measured temperature (the accuracy of temperature measurements is of $\pm 0.1 \mathrm{~K}$ ). The thermopower was measured by means of small temperature gradient into the liquid sample. The measurements were carried out in the following manner. The sample of liquid aluminium was $55 \mathrm{~mm}$ long. To suppress convection, the surface temperature of molten aluminium was kept at a temperature higher by about $3 \mathrm{~K}$ than the bottom temperature. Since the thermopower is derived from Eq. (1), we used the measured electrical resistivity values to calculate it. The resistivity was measured by a standard DC two-probe technique. The DC electrical resistivity measurements are made according to the following procedure: high-alloyed stainless steel electrodes are immersed into the melted metal. The electrode material does not react with the melt. The distance between the electrodes is $30 \mathrm{~mm}$ and the electrodes lateral surface area is the same for both immersion depths. Connection cables of large section and electrical resistance less than the resistance of the melt were used. To have a better accuracy, we used the indirect measurement method of electrical current, across the melt. Thus, the voltage level on calibrated shunt is measured by a valve voltmeter, type EO302, with an accuracy of $\pm 0.1 \mathrm{mV}$.

A power supplier of a variable output voltage and a rectifier bridge obtain the current across electrodes.

A magnetostrictive transducer and an ultrasound generator were used to generate continuous longitudinal waves into the liquid sample. We preferred to introduce the ultrasound waves through the bottom of the crucible. With this arrangement, there is no barrier between the ultrasound source and the melted metal. The stepped stainless steel horn was used to transmit the ultrasound to the melt and it is completely resistant to ultrasonic erosion. Typical operating parameters were frequency $f=20.338 \mathrm{kHz}$ and the nominal input power $600 \mathrm{~W}$. The acoustic power dissipated by ultrasonic probe in $1000 \mathrm{ml}$ deionized water at ambient temperature and pressure as a function of electrical input power was determined by calorimetry. These data were used to allow selection of the appropriate input power to give constant transmitted power. 
The distance between the electrodes and the horn was selected as $h_{1}=0.35 \lambda$ and $h_{2}=0.75 \lambda$, where $\lambda$ is the ultrasound wavelength in the stainless steel horn [11].

From the measured voltage, random and systematic errors associated with our measuring technique and equipment is unlikely to produce a standard deviation larger than $0.15 \mu \mathrm{V} / \mathrm{K}$.

\section{Results and discussions}

In this section we provide a qualitative approach to the ultrasound field influence on thermopower of liquid aluminium.

At present, close similitude might be inferred between the structures of metallic crystals and their melts, though the possibility of extended effects in the crystal in their melts affects transport coefficients. On melting, the loss of long-range correlation of positions and the general increase in positional disorder may be generally expected to increase the conduction electrons scattering. The vibration amplitudes of each atom around mean position also increase. Finally, the Fermi level of the electrons may change as a consequence of the volume change. However, these transport properties prove to be not very sensitive to structural changes on melting. Closer characterization of the Fermi surface of crystalline and molten metals might help to explain the changes of electronic properties on the basis that a liquid metal has "no long-range order", therefore the Fermi surface should be spherical and the relaxation time isotropic. As to the molten metals, which can be adequately represented as quasicrystalline, theories referred to above can be regarded as giving at least a first approximation.

In order to be able to describe the probable processes in the system liquid metal-ultrasonic field, we had to use the model potentials of simple liquid metals. These theories are based on the assumption that each ion acts upon the conduction electrons through a local pseudopotential and that the conduction electrons are nearly free.

In order to account for the effect of ultrasonic waves, the molten systems were first studied in the absence of ultrasound. The thermopower values (in various pseudopotential models) of liquid aluminium are given in Table $\mathbf{I}$.

\section{TABLE I}

Thermoelectric power for liquid aluminium.

\begin{tabular}{|c|c|c|c|}
\hline$T[\mathrm{~K}]$ & $Q_{1}[\mu \mathrm{V} / \mathrm{K}]$ & $Q_{2}[\mu \mathrm{V} / \mathrm{K}]$ & $Q_{\exp }[\mu \mathrm{V} / \mathrm{K}]$ \\
\hline 976 & 1.87 & 1.23 & 2.10 \\
\hline \multicolumn{4}{|c|}{$\begin{array}{l}Q_{1} \text { - using effective electron mass in nearly } \\
\text { free-electron theory }[7] ;\end{array}$} \\
\hline
\end{tabular}


We take the values of $Q$ for molten aluminium (in absence of ultrasound) calculated by Wang and So [7] who consider both the nonlocality and the energy independence of the model pseudopotential and also the effective mass correction. It should be noted that Wang and So introduced a theoretical electronic pseudopotential where all the liquid metal ions are considered to form a periodic lattice. There is a discrepancy between the values calculated by Wang and So [7] and experimental values. In the same silent conditions, using the value given by Heine and Abarenkov [3] who consider the nonlocal and dependent energy potential, the agreement between theory and experiment for the thermopower is largely satisfactory.

Figure 1a and $b$ shows the dependence of the liquid aluminium thermopower on the temperature in two cases: with or without ultrasound presence in the liquid metal mass and in various experimental conditions. It can be noticed that the thermopower values are smaller in the presence of ultrasound. We explain this decrease by the relationship between the two electronic transport coefficients (electrical resistivity and thermopower) [7] and using the electron-ion interaction. In a liquid metal, the presence of both an electrical field and a temperature gradient causes a strong perturbation of the electron system, so that the energetically and ionic charged particles (electrons and ions) move orderly yielding energetic and ionic charged flows. During the ultrasound waves propagation through liquid metal, specific losses appear because of the existence of nearly free-electron gas in metal. The wave propagation produces the modification of the short-range ordered structure, which further involves local Fermi energy modification and redistribution in conduction-electrons system. As a consequence of this phenomenon, an electric current appears between those regions in which the ultrasound wave produces the compression and the other regions in which the medium rarefaction takes place. Moving the charged particles is more difficult and current density is decreased,
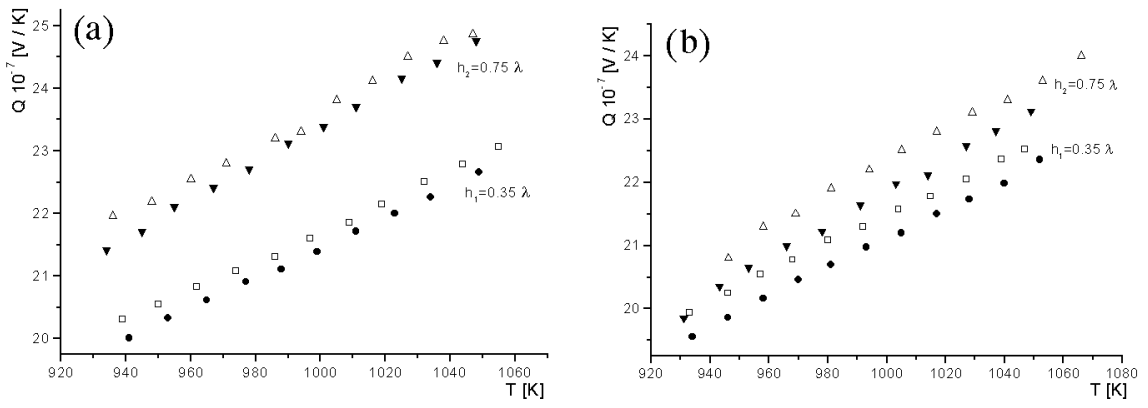

Fig. 1. Temperature dependence of the liquid aluminium thermopower as function of the electrode distance from the horn $h_{1}=0.35 \lambda$ and $h_{2}=0.75 \lambda$ in the absence (empty signs) and presence (full signs) of ultrasound. The voltage level on electrodes is: (a) for $U=2 \mathrm{~V}$ and (b) for $U=2.5 \mathrm{~V}$. 
explaining the smaller thermopower values.In addition, this redistribution mechanism will involve the relaxation process, because the equalizing of local Fermi energies is not accompanied by the compensation of the whole excess of electrical charge.
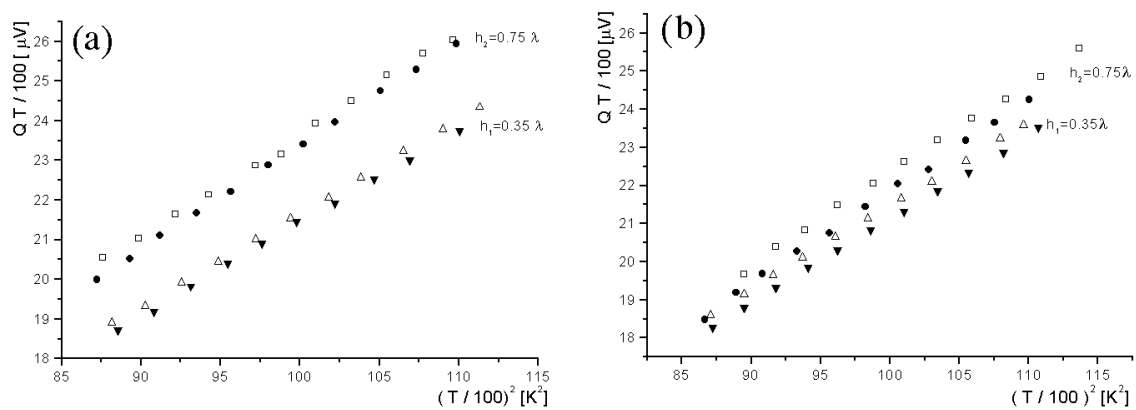

Fig. 2. Dependence $Q T / 100=f\left[(T / 100)^{2}\right]$ of liquid aluminium as a function of the electrode distance from the horn $h_{1}=35 \lambda$ and $h_{2}=0.75 \lambda$ in the absence (empty signs) and presence (full signs) of ultrasound. The voltage level on electrodes is: (a) for $U=2 \mathrm{~V}$ and (b) for $U=2.5 \mathrm{~V}$.

Figure $2 \mathrm{a}$ and $\mathrm{b}$ shows the functional dependence $Q T / 100=f\left[(T / 100)^{2}\right]$ for two sets of experimental data. The obtained dependences are absolutely linear in the chosen temperature range. These experimental data confirm the dependence $Q=-A / T+B T$. The constants are numerically computed by statistical methods.

The regression lines are given in Table II.

TABLE II

Regression lines for functional dependencies $Q=-A / T+B T$.

\begin{tabular}{c|c|c}
\hline \multirow{2}{*}{} & \multicolumn{2}{|c}{$h_{1}=0.35 \lambda$} \\
\cline { 2 - 3 } & Without ultrasound & With ultrasound \\
\hline$U=2 \mathrm{~V}$ & $-194.24 / T+2.36 \times 10^{-3} T$ & $-201.75 / T+2.37 \times 10^{-3} T$ \\
\hline$U=2.5 \mathrm{~V}$ & $-43.77 / T+2.19 \times 10^{-3} T$ & $-91.24 / T+2.20 \times 10^{-3} T$ \\
\hline & \multicolumn{2}{|c}{$h_{2}=0.75 \lambda$} \\
\hline$U=2 \mathrm{~V}$ & $-197.9 / T+2.56 \times 10^{-3} T$ & $-297.47 / T+2.63 \times 10^{-3} T$ \\
\hline$U=2.5 \mathrm{~V}$ & $-160.8 / T+2.39 \times 10^{-3} T$ & $-237.91 / T+2.42 \times 10^{-3} T$
\end{tabular}

The specific mechanism of ultrasound propagation affects the transport phenomenon. It is very likely that the decrease in thermopower under the action of ultrasound may be basically accounted for by the coupling between the ultrasound wave and conduction electrons and by the electron-ion interactions. Scattering electron-ion processes cause the decrease in $Q$ and consequently the mean free path of electrons is limited through the "ions lattice" deformed under the action 
of the ultrasonic field. At the same time, we have appreciated the modification of the Fermi surface energy. When $T \geq \Theta(\Theta$ is the Debye characteristic temperature), the maximum amount of energy $k \Theta$ that an electron can gain or lose in a collision is less than the thickness $k T$ of the thermal layer at the Fermi surface, and the effective mean free path becomes the same for both electrical and thermal conduction. The ultrasonic wave propagation produces the modification of the short-range ordered structure and involves the local Fermi energy modification, hence redistribution in conduction-electrons system. As a consequence of this phenomenon, an electric current appears between those regions where ultrasound produces the compression and those regions where medium rarefaction takes place. The moving of the charged particles is more difficult and current density decreases while thermopower values are smaller. We may conclude that all scattering processes are effectively contributing to the heat flow limitation in the molten metals.

The effect of convection arises from a temperature gradient in a liquid sample and brings about overestimation of thermopower values. To suppress steady convection, the surface temperature of molten sample was kept at a temperature higher by about $3 \mathrm{~K}$ than its bottom temperature, as mentioned in Sec. 2. This temperature difference was confirmed by thermocouples in each experiment. During the propagation of high-energy ultrasounds through the melt, acoustic flows are present [13]. In this study, the effect of the ultrasound absorption is neglected according to Beyer and Coppens [14] and Gitis and Mikhailov [15], so the temperature rise of the molten caused by this effect has been eliminated. In the same way, the temperature difference of about $3 \mathrm{~K}$ between crucible ends was maintained even in the ultrasonic field presence and the continuous mixing process of the liquid metal mass was operative due to acoustic flow. This process induces molten thermal gradient decreasing, as a result of the temperature equalization in the liquid mass. The decrease in the thermal gradient influences both electrical and thermal conduction of the sample.

The effect of the long-range external electric field, namely the drift motion, is simultaneous with the effect of the mixing process, namely a disorder of conduction electron motion. This process can be understood as a current density decrease in molten mass that induces decrease in thermopower. It is clearly demonstrated $[16,17]$ that the acoustic flows are to be associated with the ultrasound absorption, whatever its nature might be. Accurate measurements of the ultrasound propagation in liquid metals developed slowly, because of technical problems associated with getting the ultrasound into the melted metal. At present the accuracy of sonic measurements is reasonable taking into account the difficulties of experiments. However, a number of studies showed that the absorption coefficient is quite small for the liquid metals $[14,15]$ and then only the acoustic flows affected the thermopower. 
We suggest that the relationship between thermopower and temperature given by Eq. (2) represents available means for separating the thermopower of aluminium into its two component parts. The term proportional with $1 / T$ characterizes the electron-ion collisions, being associated with the probability of the electron transition, from one state to another. The term proportional with $T$ is associated with the spatial redistribution process of the conduction-electrons system in the temperature gradient field. From the analysis of the values of $A$ and $B$ constants obtained in silent and sonication conditions, it results that the redistribution of conduction electrons system in thermal field has no determined contribution to decrease in $Q$ and that the electron-ion collision and the probability of transition from a state to another becomes more important in ultrasonic field and affects the $Q$ values.

As often happens, this investigation has tended to raise new questions rather than answer the old ones. One new question is the problem of cavitation and its microstreaming effect. The effect of ultrasound increases with increasing power, but not indefinitely since there is an optimum value beyond which the effect diminishes. The acoustic amplitude affects the maximum bubble size attained during the rarefaction phase and this in turn affects both the time required for bubble collapse and the resulting collapse intensity. Cavitation intensity is maximized when the collapse of the bubble coincides with the peak in the compressive cycle, for which the collapse time is equal to half the period of oscillation. There is a peak in the cavitation intensity due to the effect of power on maximum cavity size attained during rarefaction; increasing cavity size leads to increased cavitation intensity provided the collapse time does not exceed half the period of ultrasound.

The resonant condition, which produces the maximum effect of sonication, must be a function of power as well as of frequency. As far as frequency is concerned, this will affect the strength of cavitational collapse, which will be maximized when the frequency coincides with the resonant frequency of the cavitation nuclei.

When $20.338 \mathrm{kHz}$ high-intensity ultrasound was applied to the molten system, a mixing of the melted metal close to the electrode due to cavitation was produced. Near the solid surface, cumulative jets can be generated and the diffusion layer is thinned owing to enhanced mass transport resulting from microstreaming. Church [18] confirms that, when microstreaming is present, it may affect the bubble size distribution and therefore the cavitational effects.

In our experiment, these optimum conditions in cavitation were studied in deionized water at ambient temperature. The ultrasonic treatment of liquid metals differed essentially from that of aqueous solutions and organic liquids. This is due to the different nature of cavitation nuclei and, hence different conditions required for the initiation and development of acoustic cavitation. Only fine solid particles (mainly oxides, e.g. $\mathrm{Al}_{2} \mathrm{O}_{3}$ in aluminium melt) can act as cavitation nuclei in metallic melts [19]. At the same time, the temperature of experimental system 
is strongly increasing and, as the molten metals feature light opacity, the cavitation cannot be studied directly. Thus, the cavitation effect is affected. Sarveswara Rao et al. [20] report an ultrasound pronounced effect at lower temperatures by improved cavitation, taking into account two different temperatures in the range $288 \mathrm{~K}$ to $318 \mathrm{~K}$. In conclusion, additional experiments at different acoustic power are necessary to check the dependence of electrical change on the acoustic power.

The experimental conditions together with the analysis of the acoustic flows and the cavitation effect permit to predict that at high temperature, all these factors equally contribute to the thermopower decrease in liquid metals exposed to ultrasound.

The liquid metals possess some sort of internal arrangement of atom [7]. It may be speculated that during the ultrasound propagation through the liquid metal the deformation of "ionic lattice" takes place. This process modifies the state energy of conduction electrons and they return to the equilibrium state by successive collisions with the ions associated to the pseudolattice. These collision processes contribute to the electrons disordered movement and produce a variation of electron distribution. This suggests a relaxation process, when electrons try to achieve a local equilibrium distribution and an energetic dissipation phenomenon occurs. Its may also reflect the lower thermopower values.

The linear dependence $Q=Q(T)$ for $h_{1}=0.35 \lambda$ distance from the ultrasonic source is characterized by smaller experimental dispersion values. We may even say that the linear dependence $Q=Q(T)$ is better reproduced while the applied electric field increases. The decreasing dispersion values of $Q$ for smaller distance of electrodes from ultrasonic horn may be explained by a degassing effect of the liquid metal under the action of ultrasound [21]. Consequently, the melt surface layers are contaminated with these gas bubbles that disturbed the experimental conditions. For $h_{2}=0.75 \lambda$ distance from horn, the experimental dispersion values are higher. The experimental results showed that the effect of sonication increased as the ultrasonic probe approached the electrodes. The thermopower decreases with decrease in horn to electrode distance. There must in fact be a critical distance, which represents the maximum extent of the effective cavitation field. Chivate and Pandit [22] showed that the effect of cavitation (namely maximum pressure impulse) increased approximately exponentially with decreasing distance. The pressure intensity developed by the oscillating horn tip decreases exponentially as it moves away from the horn tip, so that the energy available to overcome the cavitation threshold is lowered. For distance $h_{2}=0.75 \lambda$ from horn, fewer cavities are formed. The thermopower values are higher than those measured at $h_{1}=0.35 \lambda$ distance from horn.

The melt metal bath has not been protected from oxidation and the immersion depths of electrodes are necessary to reduce as much as possible the influence of aluminium oxide layer formed at the melted surface. 


\section{Conclusions}

In this study the influence of ultrasound on the liquid aluminium thermopower is investigated.

The decrease in $Q$ values in the presence of ultrasonic field has been explained by both electron-ion collision and scattering of conduction electron processes. The ultrasound propagation through the liquid metal induces its "ionic lattice" deformation; therefore a drift movement perturbation of electrons appears. The electrons return to their equilibrium state due to the electron-ion interaction, the result being exactly the decrease in the liquid metal thermopower. At the same time, due to the acoustic flows the temperature equalization between different zones of the liquid metal takes place and the melt temperature gradient is decreasing. The smaller values of temperature gradient determine lower $Q$ values of liquid aluminium. The cavitation effect is considered.

Finally, it should be pointed out that discussions on these interesting differences in the behavior under sonication conditions are still far from definitive.

\section{References}

[1] J.M. Ziman, Philos. Mag. 6, 1013 (1961).

[2] J.M. Ziman, in: The Properties of Liquid Metals, Eds. P.D. Adams, H.A. Davies, S.G. Epstein, Taylor and Francis, London 1967, p. 551.

[3] V. Heine, I. Abarenkov, Philos. Mag. 9, 451 (1964).

[4] A.E.O. Animalu, V. Heine, Philos. Mag. 12, 1249 (1965).

[5] N.W. Ashcroft, J. Phys. C 1, 232 (1968).

[6] N.W. Ashcroft, N.D. Mermins, Solid State Physics, Saunders, Philadelphia 1988.

[7] S. Wang, C.B. So, J. Phys. F, Met. Phys. 7, 1439 (1977).

[8] W. Nicodemi, Metalurgia, EDIMET, Milano 1990, p. 619.

[9] V.L. Moruzzi, J.F. Janak, A.R. Williams, Calculated Electronic Properties of Metals, Pergamon, New York 1978.

[10] I. Takamichi, R.I.L. Guthrie, The Physical Properties of Liquid Metals, 2nd ed., Clarendon Press, Oxford Science Publication, England 1993.

[11] L. Moraru, Czech. J. Phys. 49, 253 (1999).

[12] C.H. Woo, S. Wang, M. Matsuura, J. Phys. F, Met. Phys. 5, 1849 (1975).

[13] O.V. Abramov, Ultrasound in Liquid and Solid Metals, Russian Academy of Sciences, Moscow 1993 (in English).

[14] R.T. Beyer, A.B. Coppens, in: Proc. Int. Congress on Acoustics 5th, Ed. W.P. Mason, Academic Press, Liege 1965, p. 231.

[15] M.B. Gitis, I.G. Mikhailov, Sov. Phys. Acoust. 12, 14 (1996) (in English).

[16] Y. Tomita, M. Tsubota, K. Nagane, N. An-naka, J. Appl. Phys. 88, 5993 (2000).

[17] P.A. Deymier, J.O. Vasseur, A. Khelif, B. Djafari-Rouhani, L. Dobrzynski, S. Raghavan, J. Appl. Phys. 88, 6821 (2000). 
[18] C.C. Church, J. Acoust. Soc. Am. 84, 1758 (1988).

[19] G.I. Eskin, Ultrason. Sonochem. 2, S137 (1995).

[20] K. Sarveswara Rao, K.L. Narayana, K.M. Swamy, J.S. Murty, Metall. Mater. Trans. B 28, 721 (1977).

[21] L. Moraru, C. Gheorghies, in: Proc. Natl. Physics Conf., Baia Mare (Romania) 1995, Inst. of Atomic Physics, Bucuresti 1995, p. 69 (in Romanian).

[22] M.M. Chivate, A.B. Pandit, Ultrason. Sonochem. 1, 819 (1995). 